\title{
Substrate activity screening (SAS): a general procedure for the preparation and screening of a fragment-based non-peptidic protease substrate library for inhibitor discovery
}

\author{
Andrew W Patterson ${ }^{1}$, Warren J L Wood ${ }^{1,2}$, \& Jonathan A Ellman ${ }^{1}$ \\ ${ }^{1}$ Department of Chemistry, University of California, Berkeley, CA 94720, USA. ${ }^{2}$ Present address: Oregon Health and Science University, Portland, OR 97239, USA.
Correspondence should be addressed to J.A.E. (jellman@uclink.berkeley.edu).
}

Published online 8 March 2007; doi:10.1038/nprot.2007.28

Substrate activity screening (SAS) is a fragment-based method for the rapid development of novel substrates and their conversion into non-peptidic inhibitors of Cys and Ser proteases. The method consists of three steps: (i) a library of $\mathrm{N}$-acyl aminocoumarins with diverse, low-molecular-weight $\mathrm{N}$-acyl groups is screened to identify protease substrates using a simple fluorescence-based assay; (ii) the identified $\mathrm{N}$-acyl aminocoumarin substrates are optimized by rapid analog synthesis and evaluation; and (iii) the optimized substrates are converted into inhibitors by direct replacement of the aminocoumarin with known mechanism-based pharmacophores. This protocol describes a general procedure for the solid-phase synthesis of a library of $\mathrm{N}$-acyl aminocoumarin substrates and the screening procedure to identify weak binding substrates.

\section{INTRODUCTION}

We have developed the first substrate-based fragment identification method, called 'substrate activity screening' (SAS) ${ }^{1-3}$. This method addresses two key challenges in fragment-based screening: (i) the accurate and efficient identification of weak binding fragments and (ii) the rapid optimization of the initial weak binding fragments into higher-affinity compounds ${ }^{4,5}$. In this method (Fig. 1), a library of $\mathrm{N}$-acyl aminocoumarins with diverse, low-molecular-weight $N$-acyl fragments is prepared and then, in a first step, the library is screened to identify protease substrates using a single-step, highthroughput fluorescence-based assay. The substrate-based screening method in Step 1 has important attributes in detecting weak binding fragments in addition to being high throughput and straightforward to perform. False positives as a result of aggregation, protein precipitation or non-specific binding seen in traditional inhibitor screens are not observed. In fact, in the present approach, both an active enzyme and productive active-site binding are required for the protease-catalyzed amide bond hydrolysis to occur and for the subsequent release of the fluorescent coumarin group to be observed. In addition, in contrast to direct binding assays and traditional inhibitor screens, catalytic substrate turnover results in signal amplification, and therefore even very weak substrates can be identified at concentrations where only minimal binding to the enzyme occurs. The second and third steps (Fig. 1), which are not covered in this protocol, provide a strategy for systematically and efficiently optimizing substrates incorporating weak binding fragments into high-affinity inhibitors. In the second step, the activity of the substrates is rapidly optimized by the straightforward solidphase synthesis and subsequent assay of focused libraries of substrate analogs. The third step then builds upon a key attribute of this mechanism-based substrate screen, namely, that the $N$-acyl aminocoumarin must be precisely oriented in the active site to enable productive substrate cleavage and, therefore, that the aminocoumarin can be replaced with mechanism-based pharmacophores to provide protease inhibitors directly. The choice and the versatility of pharmacophores allow reversible or irreversible inhibitors to be obtained rapidly once efficient substrates are identified.

We have successfully applied the SAS method to both Cys and Ser protease targets to identify novel, low-molecular-weight substrates and have subsequently optimized and converted the substrates into potent, non-peptidic inhibitors ${ }^{1-3}$. The SAS method was initially successfully applied to the identification of two distinct classes of novel, non-peptidic inhibitors with nanomolar affinity to cathepsin $\mathrm{S}$, which is an enzyme implicated in a number of autoinflammatory diseases (Fig. 2) ${ }^{6}$. In the first step, a diverse library of $\mathrm{N}$-acyl 7-amino-4-methylcoumarin acetic acid (AMCA) derivatives was screened to identify multiple distinct substrate classes. In the second step, substrate optimization by rapid analog synthesis and

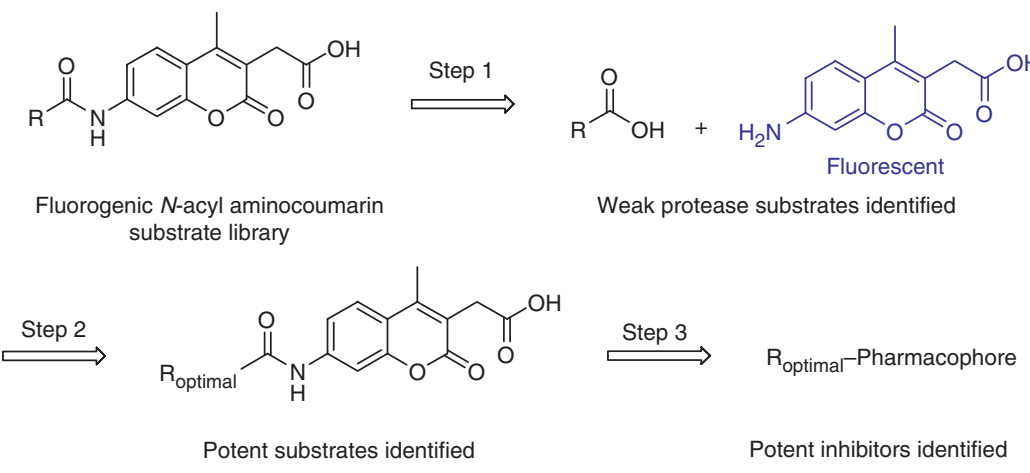

Figure 1 | Outline of the substrate activity screening (SAS) method. 
evaluation resulted in substrates with more than 8,000 -fold increases in cleavage efficiency relative to the initial substrates. Direct conversion of the substrates into inhibitors was then accomplished simply by replacing the aminocoumarin with a hydrogen atom to introduce the aldehyde mechanism-based pharmacophore ${ }^{1}$. The novel inhibitors that resulted from this process are distinct from previously reported inhibitors of cathepsin $S^{6,7}$. Using the SAS method and guided by the crystal structure of a triazole inhibitor bound to cathepsin $\mathrm{S}$, we increased the activity of the 1,4-disubstituted-1,2,3-triazole-based substrates tenfold. Conversion into inhibitors with the clinically validated nitrile pharmacophore ${ }^{8}$ provided potent inhibitors $\left(K_{\mathrm{i}}=\right.$ $15 \mathrm{nM}$ for cathepsin S) with more than 1,000 -fold selectivity over the closely related cathepsins $\mathrm{B}, \mathrm{K}$ and $\mathrm{L}^{2}$. This method is also applicable to Ser proteases, as demonstrated for the prototypical Ser protease chymotrypsin. Specifically, a novel, potent and completely non-peptidic irreversible inhibitor of chymotrypsin was discovered that had excellent selectivity over the related Ser proteases, namely, cathepsin G, elastase and tryp $\sin ^{3}$. These results demonstrate that potent and selective nonpeptidic inhibitors of distinct classes of proteases can be obtained using this substrate-based method for fragment identification and optimization.

In this protocol, the synthesis of a diverse, low-molecular-weight $\mathrm{N}$-acyl aminocoumarin library and the screening procedure against a protease target is described. A library of $N$-acyl aminocoumarins (5) can be prepared according to the procedure shown in Figure 3. In the first step, 9-fluorenylmethyloxycarbonyl (Fmoc)-protected AMCA (Fmoc-AMCA, 7), which can easily be synthesized according to the method shown in Figure 4, is loaded onto Wang resin to provide the support-bound aminocoumarin 2. The AMCA fluorogenic group was chosen because it is bifunctional and therefore suitable for rapid solid-phase synthesis. In addition, the AMCA coumarin showed greater sensitivity and higher solubility than other coumarins in the substrate assay. Wang resin was chosen as

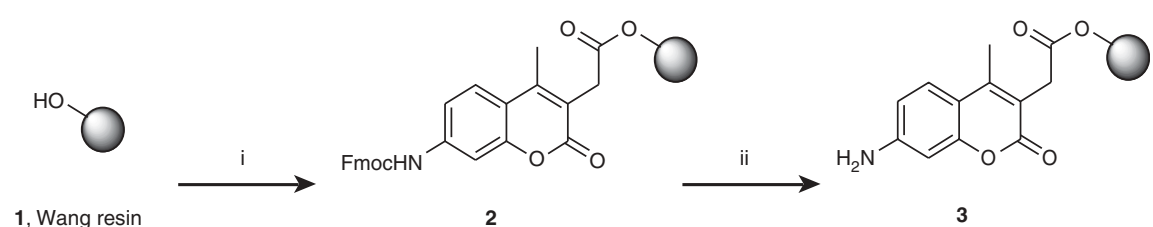

1, Wang resin

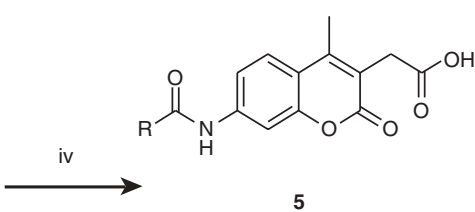

Figure 3 | Scheme for the synthesis of a diverse substrate library (5). Reaction conditions: (i) FmocAMCA (7), 2,6-dichlorobenzoyl chloride, pyridine, DMF; (ii) piperidine, DMF; (iii) $\mathrm{RCO}_{2} \mathrm{H}$, HATU, 2,4,6collidine, DMF; (iv) $9: 1 \mathrm{CH}_{2} \mathrm{Cl}_{2}:\left(95 \% \mathrm{CF}_{3} \mathrm{CO}_{2} \mathrm{H}, 2.5 \% \mathrm{H}_{2} 0,2.5 \%\right.$ triisopropylsilane). the solid-support because it allows for easy access to substrates with a free carboxylic acid. In general, however, any aminocoumarin and solid-support could be chosen. In the second step of the $N$-acyl aminocoumarin synthesis, the Fmoc group can be removed to liberate aminocoumarin 3 , which can then be acylated with diverse carboxylic acids and further derivatized, if desired, to yield the support-bound coumarin 4 . The carboxylic acid inputs should be of low molecular weight (less than $300 \mathrm{Da}$ ), compatible with the solid-phase synthetic scheme, and either commercially available or easily obtained using synthetic methods. The general procedure below is for the synthesis of one library member. To synthesize the full library of $\mathrm{N}$-acyl aminocoumarin substrates, Fmoc-AMCAWang resin (2) should be prepared on a larger scale and split into portions for each substrate to be synthesized. Note that Steps 31-54 in the procedure can easily be conducted in parallel to expedite synthesis. Following acidic cleavage from support and purification, a diverse library of $\mathrm{N}$-acyl aminocoumarins (5) is obtained. The screening procedure described is for cathepsin $\mathrm{S}$ but should be easily modifiable to be applicable to any Cys or Ser protease because peptidyl coumarin fluorogenic substrates have been shown to be efficiently cleaved by a very large number of diverse Cys and Ser proteases ${ }^{9}$. In designing the substrate assay, a number of items are of great importance. First, an appropriate buffer must be chosen. This will usually be the buffer that is optimal for known peptidic substrates, but modification may be needed owing to $N$-acyl aminocoumarin solubility. Second, the proper substrate concentration in the assay must be identified. In general, the highest substrate concentration for which none of the substrates precipitates/ aggregates is best for identifying the greatest 
number of weak binding substrates. This concentration may vary based on the buffer chosen and average solubility of the library members. In addition, a small peptidic $N$-acyl aminocoumarin substrate, either a mono- or dipeptidic substrate, that is cleaved by the protease under the assay conditions should be identified and included in each assay as a positive control. For cathepsin S, the positive control was Bz-Gly-AMCA, which served as an ideal substrate because complete substrate consumption is not observed over the course of the assay. Finally, a proper concentration of protease must be chosen. For cathepsin $\mathrm{S}$ and chymotrypsin, the substrate library was first screened at an enzyme concentration 500- to 1,000-fold lower than that of the substrate, but for less active enzymes, a higher concentration of enzyme might be required.

This protocol describes the procedure for assaying $19 \mathrm{~N}$-acyl aminocoumarins in duplicate against cathepsin S (one 96-well plate), which can be repeated or conducted in parallel to assay a larger $N$-acyl aminocoumarin library. The second and third steps of the SAS method (Fig. 1) are not explicitly described in this protocol, as optimization of lead substrates and inhibitor synthesis will vary greatly depending on the structure of the lead substrate. After screening the diverse library, optimization of weak substrates into potent substrates (step 2 in Fig. 1) can be performed before converting substrates into inhibitors because transition-state theory for enzyme-catalyzed reactions predicts that the inhibitory activity $\left(K_{\mathrm{i}}\right)$ of mechanism-based inhibitors can be correlated with the

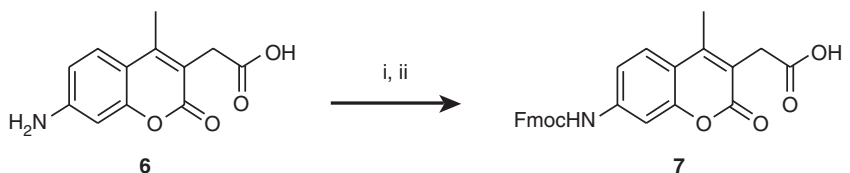

Figure 4 | Scheme for the synthesis of Fmoc-AMCA (7) from AMCA (6). Reaction conditions: (i) $\mathrm{TMSCl}, i-\mathrm{Pr}_{2} \mathrm{EtN}, \mathrm{CH}_{2} \mathrm{Cl}_{2}$; (ii) Fmoc- $\mathrm{Cl}, \mathrm{CH}_{2} \mathrm{Cl}_{2}$.

inverse of the catalytic efficiency of the corresponding substrates $\left(K_{\mathrm{m}} / k_{\mathrm{cat}}\right)^{10}$. Other researchers have confirmed this correlation for peptide substrates ${ }^{11,12}$, and we have observed this correlation for non-peptidic substrates and inhibitors for both cathepsin $S$ and chymotrypsin ${ }^{1-3}$. To obtain accurate relative substrate cleavage efficiencies $\left(k_{\mathrm{cat}} / K_{\mathrm{m}}\right)$ in the substrate optimization step, assays should be conducted at substrate concentrations below the $K_{\mathrm{m}}$ of the substrates (Michaelis-Menten equation: $v=k_{\mathrm{cat}}[\mathrm{E}][\mathrm{S}] /\left(K_{\mathrm{m}}+\right.$ $[S])$. This can be accomplished by periodically determining an approximate $K_{\mathrm{m}}$ for the most potent substrate(s) in a series. In the third step of the SAS method (Fig. 1), the selection of a pharmacophore for inhibitors will depend upon the protease of interest. For example, for cathepsin $\mathrm{S}$ and other papain-family Cys proteases, aldehyde, ketone, nitrile and arylaminoethyl amide groups are documented to be viable reversible pharmacophores ${ }^{13-15}$, whereas chloromethyl ketones are documented as general irreversible inhibitor pharmacophores for X-ray crystallographic study ${ }^{16}$.

\section{MATERIALS}

\section{REAGENTS}

- Dichloromethane $\left(\mathrm{CH}_{2} \mathrm{Cl}_{2}\right.$; Fisher, cat. no. D143-4) $\triangle$ CRITICAL When specified, $\mathrm{CH}_{2} \mathrm{Cl}_{2}$ is purified by distillation over calcium hydride to remove water.

- Chlorotrimethylsilane (TMSCl; Sigma-Aldrich, cat. no. 386529)

- Diisopropylethylamine ( $i$ - $\mathrm{Pr}_{2} \mathrm{EtN}$; Fluka, cat. no. 03440) $\Delta$ CRITICAL When specified, $i-\mathrm{Pr}_{2} \mathrm{EtN}$ is purified by distillation over calcium hydride to remove water.

- Pyridine (Sigma-Aldrich, cat. no. 360570) $\Delta$ CRITICAL When specified, pyridine is purified by distillation over calcium hydride to remove water. - Calcium hydride (Aldrich, cat. no. 558257) ! CAUTION Calcium hydride is a flammable solid and is water reactive.

- AMCA (Fluka, cat. no. 08445); can be synthesized analogously to the synthesis of 7-amino-4-carbamoylmethylcoumarin (ACC) ${ }^{17}$

-9-Fluorenylmethyloxycarbonyl chloride (Fmoc-Cl; Fluka, cat. no.23185)

- Methanol (MeOH; Fisher, cat. no. A452-4)

- Diethyl ether $\left(\mathrm{Et}_{2} \mathrm{O}\right.$; Fisher, cat. no. E198-4)

-Wang resin, 100-200 mesh, 0.9-1.3 mmol g ${ }^{-1}$ (Novabiochem, cat. no. 01-64-0014)

- Peptide synthesis-grade dimethylformamide (DMF; EM Science, cat. no. DX1732-1)

-2,6-Dichlorobenzoyl chloride (Aldrich, cat. no. 187518)

- Tetrahydrofuran (THF; Fisher, cat. no. T425-4)

- Piperidine (Sigma-Aldrich, cat. no. 411027)

-O-(7-Azabenzotriazol-1-yl)-1,1,3,3,-tetramethyluronium

hexafluorophosphate (HATU; PerSeptive Biosystems, cat. no. GEN076523)

$\cdot 2,4,6$-Collidine (Aldrich, cat. no. 142387)

- Trifluoroacetic acid (TFA; Acros, cat. no. 13972)

- Triisopropylsilane (TIS; Aldrich, cat. no. 233781)

- Acetonitrile, HPLC grade (MeCN; Fisher, cat. no. A998-4)

-DMSO, spectrophotometric grade (Acros, cat. no. 16785-0010)

- Polyoxyethylenesorbitanmonolaurate (Tween-20; Sigma-Aldrich, cat. no. 27434-8)

-EDTA, tetrasodium salt DNase, RNase, protease free (Acros, cat. no. 32721-1000)
- DL-1,4-DTT, DNase, RNase, protease free (Acros, cat. no. 32719-0100)

- Sodium phosphate dibasic heptahydrate (EM Science, cat. no. SX0715-1)

- Sodium phosphate monobasic monohydrate (EM Science, cat.

no. SX0710-1)

- Cathepsin S, from human spleen (CalBioChem, cat. no. 219344)

- Buffers (see REAGENT SETUP)

EQUIPMENT

-100-ml 24/40 round-bottomed flask (Fisher, cat. no. 10-067C)

$\cdot 1,000$-ml side-armed flask (Fisher, cat. no. 10-180F)

-250-ml separatory funnel (Fisher, cat. no. 10-437C)

- 250-ml Erlenmeyer flask (Fisher, cat. no. 10-040F)

- 4-ml glass vial (Fisher, cat. no. 02-912-368)

-24/40 reflux condenser (VWR, cat. no. 80067-290)

-24/40 rubber septum (Aldrich, cat. no. Z10,145-1)

-12-ml polypropylene cartridges with 70-mm polyethylene frits (Applied

Separations, cat. no. 2427)

- Teflon stopcocks (Applied Separations, cat. no. 2406)

- Tygon tubing (Fisher, cat. no. 14-169-1K)

- Polypropylene plastic syringes (Fisher, $1 \mathrm{ml}$, cat. no. 14-817-25; $10 \mathrm{ml}$, cat. no. 14-817-31; $50 \mathrm{ml}$, cat. no. 14-817-40)

-18-gauge disposable syringe needles (Fisher, cat. no. 14-826-5D)

-Visiprep Solid Phase Extraction Vacuum Manifold (Supelco,

cat. no. 57250-U)

- Orbital shaker (Big Bill Mixer, Geneq. Inc., cat. no. M73625)

- Rotary evaporator (Buchimodel RE111; Buchi)

- Coors porcelain Büchner funnel (Fisher, cat. no. 10-356B)

-Whatman filter paper (Fisher, cat. no. 09-855AA)

- Reverse-phase HPLC (Agilent 1100 series)

- HPLC column (preparatory reverse phase): C18, $21.4 \times 250 \mathrm{~mm}$ (Varian, cat. no. R0080220C5) (see EQUIPMENT SETUP)

- HPLC injection needle (Varian, cat. no. HM90134)

-25-mm, 0.45- $\mu \mathrm{m}$ filter (Fisher, cat. no. SLHN025)

- Milli-Q PF Plus water deionizing purification system (Millipore)

- 50-ml centrifuge tubes (Fisher, cat. no. 05-539-8)

- 15 -ml centrifuge tubes (Fisher, cat. no. 05-539-12)

- Freeze-dry system (Labconco Corporation, cat. no. 7670020) 
- Microplate spectrofluorometer equipped with a temperature regulator (SpectraMax Gemini XPS instrument; Molecular Devices) (see EQUIPMENT SETUP)

- Microfluor fluorescence 96-well microtiter black plates (Dynex, cat. no. 7205)

- Thermal adhesive sealing films for microplates (Fisher, cat. no. 05-500-32)

- Spreadsheet software with graphing capabilities (e.g., Microsoft Excel)

- Disposable pipette tips (Fisher, $10 \mu \mathrm{l}$, cat. no. 21-276A; $200 \mu \mathrm{l}$, cat. no. $02707501 ; 1,000 \mu \mathrm{l}$, cat. no. $21-197-8 \mathrm{~A}$ )

REAGENT SETUP

Buffers Buffer A: 0.001\% Tween-20 (wt/vol), 1 mM EDTA, 1 mM DTT, 100 mM sodium chloride, $100 \mathrm{mM}$ sodium phosphate $(\mathrm{pH}$ 6.1) in double-deionized water. Buffer B: buffer A containing $790 \mathrm{nM}$ cathepsin S.

\section{EQUIPMENT SETUP}

HPLC setup Configure the HPLC with a preparatory reverse-phase C18 column, a 5-ml sample loop and a UV detector set to $254 \mathrm{~nm}$. Use doubledeionized water and HPLC-grade MeCN containing 0.1\% TFA. Before using this, flush the column for 20 min with 95:5 MeCN:water at a flow rate of $8 \mathrm{ml}$ $\mathrm{min}^{-1}$. Over the next $10 \mathrm{~min}$, run a gradient in which the solvent composition changes from 95:5 to 5:95 MeCN:water and then equilibrate the column at 5:95 MeCN:water for $20 \mathrm{~min}$.

Spectrofluorometer setup Set the spectrofluorometer to an excitation wavelength of $370 \mathrm{nM}$ and an emission wavelength of $455 \mathrm{nM}$, with a cutoff of $435 \mathrm{nM}$. A cutoff wavelength helps to increase the sensitivity of the assay. Pre-incubate the spectrofluorometer at $37^{\circ} \mathrm{C}$. For spectrofluorometers not equipped for temperature regulation, the microtiter plates may be incubated externally in a properly equilibrated oven.

\section{PROCEDURE}

\section{Synthesis of Fmoc-AMCA $\bigcirc$ TIMING $22 \mathbf{h}$}

1| Fit a $100-\mathrm{ml}$ round-bottomed flask with a Teflon-coated magnetic stir bar and a reflux condenser capped with a rubber septum.

2| Remove the syringe plunger from a 1-ml plastic polypropylene syringe and cut off the top of the syringe barrel using a pair of scissors. Insert the syringe into one end of Tygon tubing and connect the other end to a vacuum manifold. Attach a disposable syringe needle to the end of the 1-ml syringe barrel and insert the needle into the septum on the reflux condenser.

3| Put the system under vacuum and flame-dry the round-bottomed flask, stir bar and reflux condenser.

4 Allow the apparatus to cool to room temperature $\left(23^{\circ} \mathrm{C}\right.$ ), remove the vacuum line and insert a nitrogen line (prepared in the same manner as in Step 2). Attach one end of Tygon tubing to a water inlet and the other end to the bottom of the condenser. Attach one end of another piece of Tygon tubing to the top of the condenser and the other end to a water outlet. Turn on the water.

5| Remove the reflux condenser quickly and add $5.0 \mathrm{~g} \mathrm{(21} \mathrm{mmol,} 1.0$ equiv.) AMCA (6) to the flask. Then reattach the reflux condenser immediately.

6| Add $35 \mathrm{ml}$ distilled $\mathrm{CH}_{2} \mathrm{Cl}_{2}$, using a needle attached to a 50-ml plastic syringe, via the septum on the condenser, to make a $0.60 \mathrm{M}$ solution. Turn the magnetic stirrer on.

7| Add $6.1 \mathrm{ml}$ (48 mmol, 2.3 equiv.) distilled TMSCl and $8.4 \mathrm{ml}$ ( $48 \mathrm{mmol}, 2.3$ equiv.) distilled $i$ - $\operatorname{Pr}_{2} \mathrm{EtN}$, using a needle attached to $10-\mathrm{ml}$ plastic syringes, via the septum on the condenser.

$\triangle$ CRITICAL STEP It is important to use freshly distilled TMSCl to obtain a high reaction yield.

8| Heat the reaction flask to $70{ }^{\circ} \mathrm{C}$ so that the reaction solution refluxes. Heat for $3 \mathrm{~h}$.

9| Remove the heating source, allow the flask to cool to room temperature and then cool the flask in an ice-water bath.

10| Remove commercially available $\mathrm{Fmoc}-\mathrm{Cl}$ from storage in a $-20{ }^{\circ} \mathrm{C}$ freezer. After the container warms to room temperature, weigh $6.2 \mathrm{~g}$ (24 mmol, 1.1 equiv.) Fmoc-Cl and add in five portions over $15 \mathrm{~min}$ to the round-bottomed flask by quickly removing the condenser for each addition.

- PAUSE POINT Remove the ice-water bath and stir the reaction mixture for $15 \mathrm{~h}$ at room temperature.

11| With vigorous stirring, add $130 \mathrm{ml}$ MeOH. Fmoc-AMCA (7) will precipitate as a white solid.

12| Filter the mixture through filter paper in a Büchner funnel attached to a 1,000-ml sidearm flask connected to vacuum.

13| Wash the solid with $100 \mathrm{ml} \mathrm{MeOH}$ in two portions and then with $750 \mathrm{~mL} \mathrm{Et}_{2} \mathrm{O}$ in four portions.

14| Collect the solid Fmoc-AMCA (7) and dry it under vacuum.

PAUSE POINT Fmoc-AMCA (7) can be stored indefinitely at $-20{ }^{\circ} \mathrm{C}$ after all solvent is removed. ? TROUBLESHOOTING

\section{Loading of Fmoc-AMCA onto Wang resin $\bigcirc$ TIMING 28-52 $h$}

15| Remove Wang resin (1) and Fmoc-AMCA (7) from the refrigerator $\left(4^{\circ} \mathrm{C}\right)$ and freezer $\left(-20{ }^{\circ} \mathrm{C}\right)$, respectively, and allow to warm to room temperature.

16 Attach a Teflon stopcock to the end of a 12-ml polypropylene cartridge, connect to the vacuum manifold and turn on the vacuum. 
PROTOCOL

17| Place $0.20 \mathrm{mmol}$ (1.0 equiv.) Wang resin (1) in the cartridge. The loading of the commercial resin we have used has ranged between 0.90 and $1.3 \mathrm{mmol} \mathrm{g}^{-1}$. Please note that this reaction has been performed on as much as $10 \mathrm{~g}$ resin.

18| To wash the resin, add $5 \mathrm{ml}$ DMF to the resin. After $2 \mathrm{~min}$, open the stopcock to remove the DMF, and re-close the stopcock. Wash the resin with DMF two more times.

19| In a vial, add $1.5 \mathrm{ml}$ DMF to $210 \mathrm{mg}(0.46 \mathrm{mmol}, 2.3$ equiv.) Fmoc-AMCA (7) to make a $0.30 \mathrm{M}$ solution. Add $62 \mu \mathrm{l}(0.76$ mmol, 3.8 equiv.) distilled pyridine to this solution and mix until Fmoc-AMCA is completely dissolved. Add this solution to the resin.

20| Slowly add (dropwise) $66 \mu \mathrm{l}(0.46 \mathrm{mmol}, 2.3$ equiv.) 2,6-dichlorobenzoyl chloride to the cartridge.

! CAUTION 2,6-Dichlorobenzoyl chloride is a corrosive liquid.

21 Remove the plunger from a 10-ml plastic syringe and insert it securely into the polypropylene cartridge. Remove the cartridge from the vacuum manifold and place cartridge horizontally on an orbital shaker.

PAUSE POINT Turn the shaker on and gently shake the cartridge for 24-48 $\mathrm{h}$.

22| Remove the plunger from the cartridge and connect the cartridge to the vacuum manifold. Turn on the vacuum.

23| Open the stopcock on the cartridge and remove the Fmoc-AMCA solution.

24| Wash the resin three times with $5 \mathrm{ml}$ DMF.

25| Wash the resin three times with $5 \mathrm{ml}$ THF.

26| Wash the resin three times with $5 \mathrm{ml} \mathrm{MeOH}$.

27| Wash the resin three times with $5 \mathrm{ml} \mathrm{THF}$.

28| Wash the resin three times with $5 \mathrm{ml} \mathrm{CH}_{2} \mathrm{Cl}_{2}$.

29| Dry the resin in the cartridge under vacuum.

PAUSE POINT Fmoc-AMCA-Wang resin (2) can be stored indefinitely at $-20^{\circ} \mathrm{C}$ after all solvent is removed.

30| Determine the loading of the resin by Fmoc quantitation ${ }^{18,19}$.

? TROUBLESHOOTING

\section{Fmoc de-protection $\bigcirc$ TIMING 30 min}

31| Remove Fmoc-AMCA-Wang resin (2) from the $-20{ }^{\circ} \mathrm{C}$ freezer and allow it to warm to room temperature.

32 Measure out 0.10 mmol Fmoc-AMCA-Wang resin (2) based on the loading determined by Fmoc quantitation and place in a cartridge equipped with a stopcock and attached to the vacuum manifold.

33| Wash the resin three times with $5 \mathrm{ml}$ DMF.

34| Make a $20 \%$ solution of piperidine with $2.0 \mathrm{ml}$ piperidine and $8.0 \mathrm{~mL}$ DMF. Add $5 \mathrm{ml}$ of this solution to the cartridge. Remove the cartridge from the vacuum manifold, insert a plunger and place the cartridge horizontally on an orbital shaker. Shake gently for 5 min.

35| Remove the plunger from the cartridge and connect the cartridge to the vacuum manifold. Turn on the vacuum and open the stopcock on the cartridge to remove the piperidine solution.

36| Wash the resin one time with $5 \mathrm{ml}$ DMF.

37| Repeat Steps 34-35.

38| Wash the AMCA-Wang resin (3) three times with $5 \mathrm{~mL} \mathrm{DMF.}$

\section{Loading carboxylic acids onto AMCA-Wang resin $\bigcirc$ TIMING $26 \mathrm{~h}$}

39| Remove HATU from refrigerator and allow it to warm to room temperature.

40| Add $1.0 \mathrm{ml}$ DMF to $0.50 \mathrm{mmol}$ (5.0 equiv.) carboxylic acid in a vial to make a $0.50 \mathrm{M}$ solution. Add $190 \mathrm{mg}(0.50 \mathrm{mmol}$, 5.0 equiv.) HATU and $66 \mu \mathrm{l}(0.50 \mathrm{mmol}, 5.0$ equiv.) 2,4,6-collidine to the solution and mix until all components are completely dissolved. 
41 Add the carboxylic acid solution to the resin. Remove the cartridge from the vacuum manifold, insert a plunger and place the cartridge horizontally on an orbital shaker.

- PAUSE POINT Shake gently for at least $12 \mathrm{~h}$. Longer reaction times $(12-48 \mathrm{~h})$ are acceptable.

42 Remove the plunger from the cartridge and connect the cartridge to the vacuum manifold. Turn on the vacuum and open the stopcock on the cartridge to remove the excess reaction solution.

43| Wash the resin one time with $5 \mathrm{ml}$ DMF.

44| Repeat Steps 39-42.

45| Wash the resin three times with $5 \mathrm{ml}$ DMF.

46| Wash the resin three times with $5 \mathrm{ml} \mathrm{THF.}$

47| Wash the resin three times with $5 \mathrm{ml} \mathrm{MeOH}$.

48| Wash the resin three times with $5 \mathrm{ml}$ THF.

49| Wash the resin three times with $5 \mathrm{ml} \mathrm{CH}_{2} \mathrm{Cl}_{2}$.

\section{Support cleavage and purification of product $\bigcirc$ TIMING $27 \mathbf{h}$}

50| Prepare a 10-ml cleavage solution of $9: 1 \mathrm{CH}_{2} \mathrm{Cl}_{2}:(95 \%$ TFA, $2.5 \%$ deionized water, $2.5 \%$ TIS) vol/vol. Add $5 \mathrm{ml}$ cleavage solution to the resin and remove the cartridge from the vacuum manifold, insert a plunger and place cartridge horizontally on an orbital shaker. Shake for 1-2 $\mathrm{h}$.

! CAUTION TFA is corrosive.

51| Remove the plunger from the cartridge and drain the cleavage solution into a $100-\mathrm{ml}$ round-bottomed flask.

52| Close stopcock and add $5 \mathrm{ml}$ cleavage solution to the resin. Wait for $2 \mathrm{~min}$ and then drain the solution from the cartridge into the round-bottomed flask.

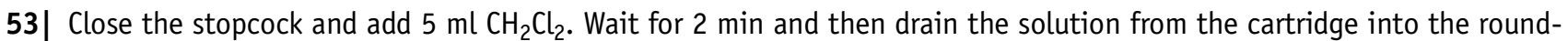
bottomed flask. Repeat the $\mathrm{CH}_{2} \mathrm{Cl}_{2}$ rinse two more times.

54 Combine the washes from Steps 51-53 and concentrate in a water bath heated to $40{ }^{\circ} \mathrm{C}$, using a rotary evaporator under reduced pressure.

PAUSE POINT The crude product can be stored at $-20{ }^{\circ} \mathrm{C}$ for at least 1 month.

55| Dissolve the crude product in the minimal amount of MeCN. Add water until the solution becomes cloudy. Add MeCN until the solution is clear. For compounds with poor solubility (compounds that require more than $5 \mathrm{ml}$ solvent to dissolve), the crude material can be dissolved in DMSO. Draw the entire solution into a $10-\mathrm{ml}$ syringe using a syringe needle. Remove the syringe needle and attach a $0.45-\mu \mathrm{m}$ syringe filter. Expel the sample through the filter into a vial to remove particulates before injecting onto the HPLC. Withdraw the entire solution into a $10 \mathrm{ml}$ polypropylene syringe using an attached HPLC needle and expel the air bubbles from the syringe.

56 Inject the sample into the injection loop of the HPLC instrument. Carry out HPLC purification using a gradient of 5:95-95:5 MeCN:water at $8 \mathrm{ml}$ per minute over $50 \mathrm{~min}$ and monitor at $254 \mathrm{~nm}$. After the run has finished, pool the fractions for each peak on the chromatogram. Conduct mass spectrometry on each peak to identify the desired product. For the most part, the peak with the greatest area is the desired product. Note that depending on the product mixture, the gradient may need to be adjusted.

57| Transfer the combined product fractions for the desired product to an appropriately sized round-bottomed flask and remove the excess MeCN and TFA by heating in a $40{ }^{\circ} \mathrm{C}$ water bath, using a rotary evaporator under reduced pressure. Be careful not to remove all the $\mathrm{H}_{2} \mathrm{O}$.

58| Transfer the remaining aqueous solution to a $50-\mathrm{ml}$ centrifuge tube and freeze the sample by placing the tube in liquid nitrogen or in a $-78{ }^{\circ} \mathrm{C}$ freezer.

59| Remove the frozen solvent using a freeze-dry system to obtain the desired $N$-acyl aminocoumarin product (5).

60| Characterize the $\mathrm{N}$-acyl aminocoumarin (5) by LCMS and ${ }^{1} \mathrm{H}$ NMR.

PAUSE POINT $N$-acyl aminocoumarins (5) can be stored indefinitely at $-20{ }^{\circ} \mathrm{C}$.

? TROUBLESHOOTING 
Preparing assay solutions for library screening $\bigcirc$ TIMING $\mathbf{1 ~ h}$

61| Dissolve purified $\mathrm{N}$-acyl aminocoumarins (5) in DMS0, dispensed using a micropipette with a disposable pipette tip, to make a $10 \mathrm{mM}$ stock solution.

! CAUTION Use appropriate gloves when handling DMSO solutions because DMSO can transport organic molecules into the body.

PAUSE POINT $N$-acyl aminocoumarin solutions can be stored indefinitely at $-20^{\circ} \mathrm{C}$.

62| Prepare buffer B: using a micropipette, dispense a portion of cathepsin $\mathrm{S}$ stock solution into $2 \mathrm{ml}$ of buffer $\mathrm{A}$ in a $15-\mathrm{ml}$ centrifuge tube. Note that cathepsin $\mathrm{S}$ solutions may be kept in an ice-water bath for approximately $1 \mathrm{~h}$ without appreciable loss of enzyme activity.

$\triangle$ CRITICAL STEP To prevent enzyme inactivation, avoid repeated freeze/thaw cycles and extended exposure to temperatures above $-78^{\circ} \mathrm{C}$.

\section{Preparing 96-well microtiter plate for assay $\bigcirc$ TIMING $\mathbf{3 0}$ min}

63| Using a micropipette, add $38 \mu$ l buffer A to two wells of the 96 -well plate (yellow wells, Fig. 5) for each $N$-acyl aminocoumarin (S1-S19) and Bz-Gly-AMCA (this peptidyl coumarin serves as a suitable positive control substrate for papainfamily Cys proteases, but a different positive control peptide substrate will likely be required for other protease classes). Note that Bz-Gly-AMCA can be prepared using standard Fmoc-based solid-phase peptide coupling strategies and should be assayed at the same concentration as the $\mathrm{N}$-acyl aminocoumarins being tested ${ }^{17}$.

64| Using a micropipette, add $2 \mu$ of each $N$-acyl aminocoumarin DMS0 solution (S1-S19 and Bz-Gly-AMCA) to two wells in the 96-well plate containing buffer A (yellow wells, Fig. 5) to make a solution that is $500 \mu \mathrm{M}$ in $\mathrm{N}$-acyl aminocoumarin.

? TROUBLESHOOTING

65| Using a micropipette, add $38 \mu \mathrm{l}$ buffer $\mathrm{B}$ to two wells of the 96 -well plate (red wells, Fig. 5 ) for each $\mathrm{N}$-acyl aminocoumarin (S1-S19) and Bz-Gly-AMCA (control).

66 Using a micropipette, add $2 \mu$ of each $\mathrm{N}$-acyl aminocoumarin DMS0 solution (S1-S19 and Bz-Gly-AMCA) to two wells in the 96-well plate containing buffer B (red wells, Fig. 5) to make a solution that is $500 \mu \mathrm{M}$ in $\mathrm{N}$-acyl aminocoumarin and $750 \mathrm{nM}$ in cathepsin S.

$\triangle$ CRITICAL STEP Ensure proper mixing between buffer solution and DMSO solution.

\section{Assay $\mathbf{N}$-acyl aminocoumarins $\bigcirc$ TIMING $6 \mathrm{~h}$}

67| Place a thermal adhesive sealing film on the microtiter plate and incubate the plate for 15 min at $37^{\circ} \mathrm{C}$.

$\triangle$ CRITICAL STEP To limit evaporation of the assay solutions, form a tight seal between the microtiter plate and the adhesive film.

68| Remove the adhesive film and perform a fluorescent reading of columns 2-11 of the 96-well plate.

69| Replace the thermal adhesive sealing film and incubate at $37{ }^{\circ} \mathrm{C}$.

70| Repeat fluorescent readings every $15 \mathrm{~min}$ for the first $2 \mathrm{~h}$ of the assay, followed by readings every 30 min until completion of the assay at $6 \mathrm{~h}$.

\section{$?$ TROUBLESHOOTING}

\section{Analyze assay data $\bigcirc$ TIMING $\mathbf{3 0}$ min}

71| Transfer the relative fluorescent unit (RFU) values corresponding to each $\mathrm{N}$-acyl aminocoumarin for each time point, with and without cathepsin S, into a spreadsheet.

72| For each $\mathrm{N}$-acyl aminocoumarin, construct a plot of RFU versus time. For active substrates, a fluorescent signal over background will be observed (Fig. 6).

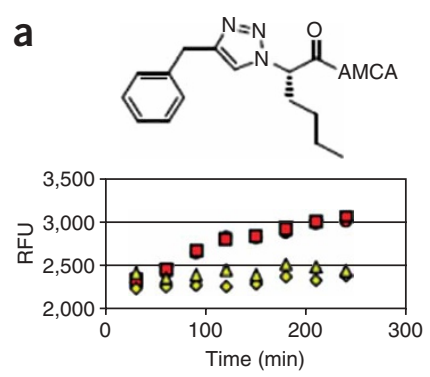

b
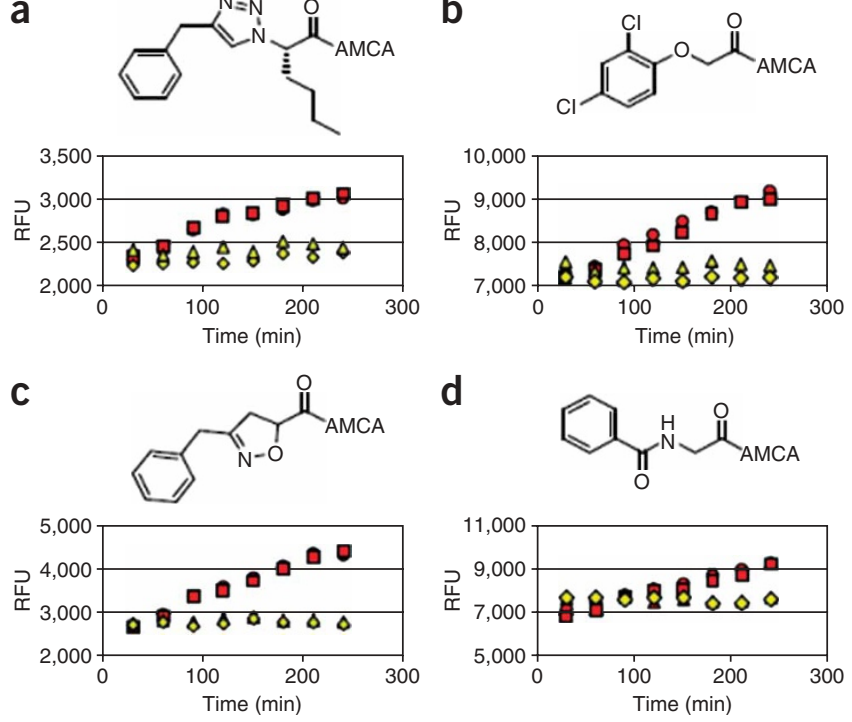

d
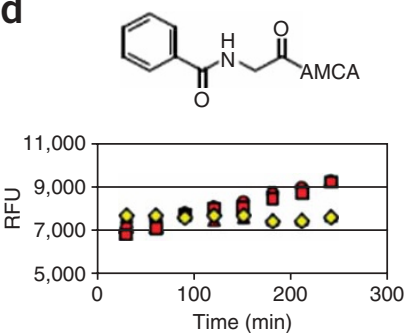

Figure 6 | Relative $k_{\mathrm{cat}} / K_{\mathrm{m}}$ plots. (a-c) Substrates of cathepsin S identified under the assay conditions; (d) control substrate Bz-Gly-AMCA. Yellow diamonds and triangles $=$ substrate in buffer $A$; red squares and circles $=$ substrate in buffer $\mathrm{B}$. 
$\Delta$ CRITICAL STEP Ensure that substrate cleavage of the positive control (Bz-Gly-AMCA) is detected (see Fig. 6 d), or else the assay is invalidated.

? TROUBLESHOOTING

\section{TIMING}

Synthesis of Fmoc-AMCA, Steps 1-4: 30 min; Steps 5-7: 30 min; Step 8: 3 h; Step 9: 1 h; Step 10: 16 h; Steps 11-14: 1 h. Loading of Fmoc-AMCA onto Wang resin, Step 15: 30 min; Steps 16-18: 10 min; Steps 19 and 20: 15 min; Step 21: 24-48 h; Steps 22-29: 35 min; Step 30: $1.5 \mathrm{~h}$.

Fmoc de-protection, Step 31: 30 min; Steps 32-38: 40 min

Loading carboxylic acids onto AMCA-Wang resin, Step 39: 30 min; Step 40: 10 min; Step 41: 12 h; Steps 42 and 43: 5 min; Step 44: $13 \mathrm{~h}$; Steps 45-49: 30 min

Support cleavage and purification of product, Step 50: $70 \mathrm{~min}$; Steps 51-53: $15 \mathrm{~min}$; Step 54: $30 \mathrm{~min}$; Step 55: $10 \mathrm{~min}$; Step 56: 50 min; Steps 57-60: $24 \mathrm{~h}$

Preparing assay solutions for library screening, Step 61: $1 \mathrm{~h}$; Step 62: $5 \mathrm{~min}$

Preparing 96-well microtiter plate for assay, Steps 63 and 64: $15 \mathrm{~min}$; Steps 65 and 66: $15 \mathrm{~min}$

Assay $\mathrm{N}$-acyl aminocoumarins, Steps 67-70: $6 \mathrm{~h}$

Analyze assay data, Steps 71 and 72: $30 \mathrm{~min}$

\section{? TROUBLESHOOTING}

Troubleshooting advice can be found in Table 1.

TABLE 1 | Troubleshooting table.

\begin{tabular}{llll}
\hline Step & Problem & Possible reasons & Possible solutions \\
\hline Library synthesis & Low yields of Fmoc-AMCA (7) & Acidic impurity of reagents, particularly & Repeat reaction with freshly distilled \\
Step 14 & TMSCl & TMSCl
\end{tabular}

Step $30 \quad$ Low yields of Fmoc-AMCA-Wang resin (2)

Step 60 Low or no product yields of $\mathrm{N}$-acyl aminocoumarins (5)

Library screening Suspension forms immediately in Step 64 assay well

Step 70 Formation of suspension in assay well between 0 and $6 \mathrm{~h}$ of incubation

Step 72

Slope of relative substrate activity is approximately 0 , but at RFU values much higher than background

Slope of relative substrate activity decreases as the assay progresses
Rapid and complete substrate consumption

Pipetting error

Substrate consumption

Enzyme inactivation owing to oxidation

Enzyme inactivation owing to smallmolecule inhibition from unknown impurity

\section{Repeat reaction with new reagent} Reagents,
chloride

Carboxylic acid is not compatible with the solid-phase method (may be piperidine or TFA sensitive)

Substrates are insoluble in this buffer at this concentration

Evaporation of buffer

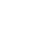


PROTOCOL

TABLE 1 | Troubleshooting table (continued).

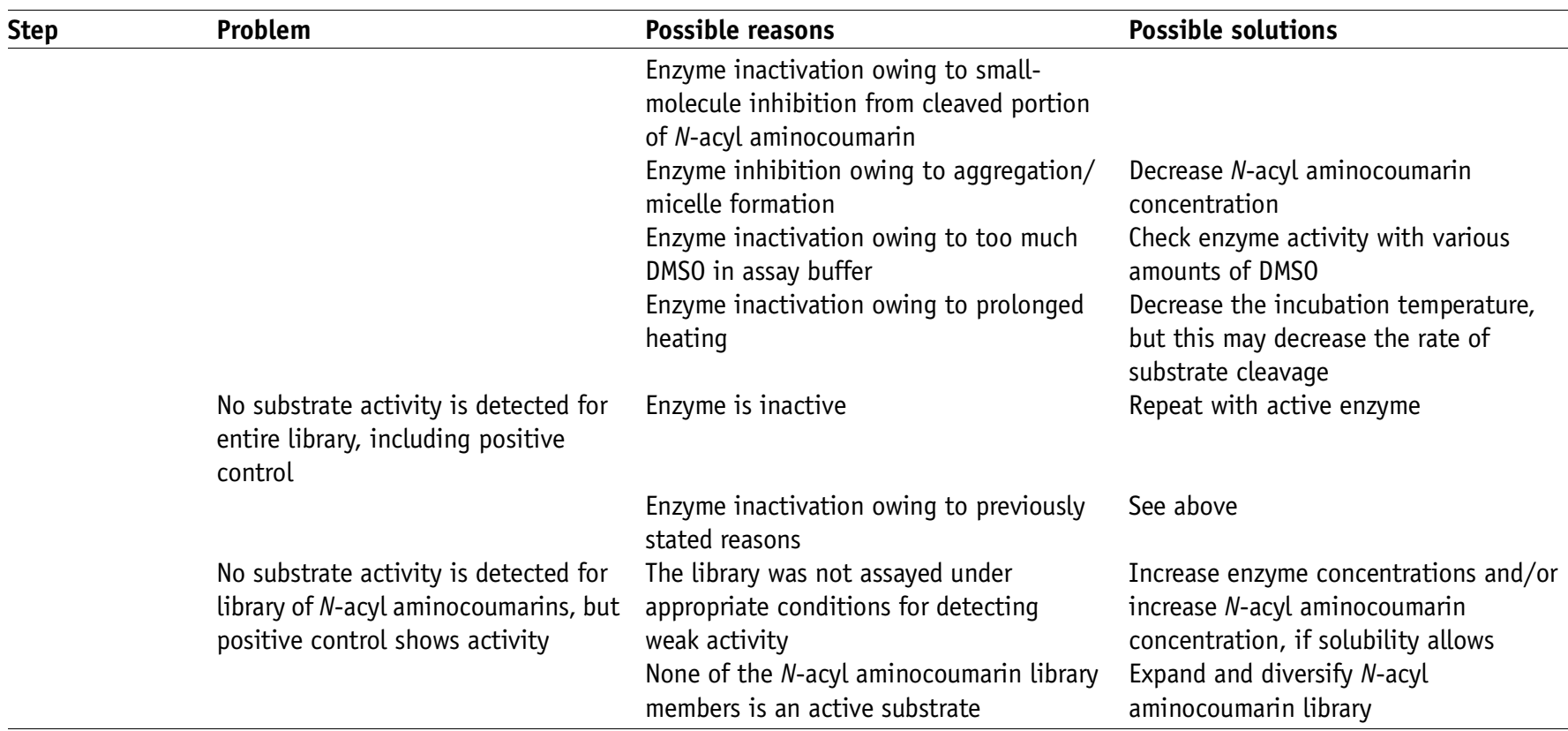

AMCA, 7-amino-4-methylcoumarin acetic acid; Fmoc, 9-fluorenylmethyloxycarbonyl; RFU, relative fluorescent unit; TFA, trifluoroacetic acid; TMSCl, chlorotrimethylsilane.

\title{
ANTICIPATED RESULTS \\ Library Synthesis \\ Typical yields
}

Typical isolated yield of Fmoc-AMCA (7) will be approximately 90\%. As determined by Fmoc quantitation, the typical loading of Fmoc-AMCA resin (2) is $0.30-0.72 \mathrm{mmol} \mathrm{g}^{-1}$. Typical yields of library members will vary depending on the carboxylic acids.

\author{
Analytical Data \\ Fmoc-AMCA (7) \\ ${ }^{1} \mathrm{H}$ NMR $\left(300 \mathrm{MHz}, \mathrm{DMSO}-\mathrm{d}_{6}\right): \delta 2.32(\mathrm{~s}, 3 \mathrm{H}), 3.55(\mathrm{~s}, 2 \mathrm{H}), 4.30(\mathrm{t}, \mathrm{J}=6.3,1 \mathrm{H}), 4.53(\mathrm{~d}, \mathrm{~J}=6.3,2 \mathrm{H}), 7.28-7.44(\mathrm{~m}, 5 \mathrm{H}), 7.51$ \\ $(\mathrm{s}, 1 \mathrm{H}), 7.64-7.77(\mathrm{~m}, 3 \mathrm{H}), 7.88(\mathrm{~d}, \mathrm{~J}=7.5,2 \mathrm{H}), 10.15(\mathrm{~s}, 1 \mathrm{H}), 12.44$ (br s, $1 \mathrm{H}) .{ }^{13} \mathrm{C} \mathrm{NMR}\left(75 \mathrm{MHz} \mathrm{CDCl}_{3}\right): \delta 15.6,33.4,47.3,66.6$, \\ $105.1,115.2,115.4,118.2,120.9,125.8,126.9,127.9,128.4,141.5,142.8,144.4,149.5,153.1,154.0,161.6,172.3$. HRMS \\ $(\mathrm{FAB}+)(\mathrm{m} / \mathrm{z})\left(\left[\mathrm{M}+\mathrm{H}^{+}\right]\right.$calcd for $\mathrm{C}_{27} \mathrm{H}_{22} \mathrm{NO}_{6}, 456.1447$, found 456.1446).
}

\section{Library Screening}

With a diverse $\mathrm{N}$-acyl aminocoumarin library of approximately 100 compounds, synthesized from small, commercially available or easily synthesized carboxylic acid precursors, we identified three distinct lead substrates for cathepsin S. Results will vary depending on the protease target and the diversity of the library.

ACKNOWLEDGMENTS The authors would like to thank the National Institutes of Health (GM54051) for support of this work. A.W.P. greatly appreciates an American Chemical Society Medicinal Chemistry Graduate Student Fellowship sponsored by Bristol-Myers Squibb.

COMPETING INTERESTS STATEMENT The authors declare that they have no competing financial interests.

Published online at http://www.natureprotocols.com Reprints and permissions information is available online at http://npg.nature.com/ reprintsandpermissions

1. Wood, W.J.L., Patterson, A.W., Tsuruoka, H., Jain, R.K. \& Ellman, J.A. Substrate activity screening: a fragment-based method for the rapid identification of nonpeptidic protease inhibitors. J. Am. Chem. Soc. 127, 15521-15527 (2005).

2. Patterson, A.W. et al. Identification of selective, nonpeptidic nitrile inhibitors of cathepsin S using the substrate activity screening method. J. Med. Chem. 49, 6298-6307 (2006).
3. Salisbury, C.M. \& Ellman, J.A. Rapid identification of potent nonpeptidic serine protease inhibitors. Chembiochem. 7, 1034-1037 (2006).

4. Erlanson, D.A., McDowell, R.S. \& O'Brien, T. Fragment-based drug discovery. J. Med. Chem. 47, 3463-3482 (2004).

5. Keseru, G.M. \& Makara, G.M. Hit discovery and hit-to-lead approaches. Drug Discov. Today 11, 741-748 (2006).

6. Link, J.0. \& Zipfel, S. Advances in cathepsin S inhibitor design. Curr. Opin. Drug Discov. Devel. 9, 471-482 (2006).

7. Leung-Toung, R. et al. Thiol proteases: inhibitors and potential therapeutic targets. Curr. Med. Chem. 13, 547-581 (2006).

8. Villhauer, E.B. et al. 1-[[(3-hydroxy-1-adamantyl)amino]acetyl]-2-cyano-(S)pyrrolidine: a potent, selective, and orally bioavailable dipeptidyl peptidase IV inhibitor with antihyperglycemic properties. J. Med. Chem. 46, 2774-2789 (2003).

9. Choe, Y. et al. Substrate profiling of cysteine proteases using a combinatorial peptide library identifies functionally unique specificities. J. Biol. Chem. 281, 12824-12832 (2006). 
10. Mader, M.M. \& Bartlett, P.A. Binding energy and catalysis: the implications for transition-state analogs and catalytic antibodies. Chem. Rev. 97, 1281-1302 (1997).

11. Thornberry, N.A. et al. A combinatorial approach for determining protease specificities: application to interleukin-1â converting enzyme (ICE). Chem. Biol. 4, 149-155 (1997).

12. Lesner, A., Kupryszewski, G. \& Rolka, K. Chromogenic substrates of bovine $\beta$-trypsin: the influence of an amino acid residue in $\mathrm{P} 1$ position on their interaction with the enzyme. Biochem. Biophys. Res. Commun. 285, 1350-1353 (2001).

13. Leung, D., Abbenante, G. \& Fairlie, D.P. Protease inhibitors: current status and future prospects. J. Med. Chem. 43, 305-341 (2000).

14. Loser, R., Schilling, K., Dimmig, E. \& Gutschow, M. Interaction of papain-like cysteine proteases with dipeptide-derived nitriles. J. Med. Chem. 48, 7688-7707 (2005).
15. Liu, H. et al. Design and synthesis of arylaminoethyl amides as noncovalent inhibitors of cathepsin S. Part 1. Bioorg. Med. Chem. Lett. 15, 4979-4984 (2005).

16. Powers, J.C., Asgian, J.L., Ekici, 0.D. \& James, K.E. Irreversible inhibitors of serine, cysteine, and threonine proteases. Chem. Rev. 102, 4639-4750 (2002).

17. Maly, D.J. et al. Expedient solid-phase synthesis of fluorogenic protease substrates using the 7-amino-4-carbamoylmethylcoumarin (ACC) fluorophore. J. Org. Chem. 67, 910-915 (2002).

18. Bunin, B.A. Fmoc quantitation. In The Combinatorial Index 5.2.2 219-220 (Academic Press, San Diego, USA, 1998).

19. Peptide synthesis protocols. In Novabiochem Catalog 3.1-3.10 (EMD Biosciences, San Diego, USA, 2004/2005). 